\title{
Polymer Nanocomposites: Synthesis, Microstructure, and Properties ${ }^{1)}$
}

Vikas Mittal

\section{1}

\section{Introduction}

Polymer-silicate nanocomposites are hybrid organic inorganic materials, in which mixing of the filler phase is achieved at the nanometer level, so that at least one dimension of the filler phase is less than $100 \mathrm{~nm}$. During recent years, these nanocomposites have generated much research interest owing to remarkable enhancements in the various composite properties at very low volume fractions [1-10]. This morphology of nanoscale dispersion of the filler phase in the polymer matrix leads to tremendous interfacial contacts of the nanoparticles with the polymer matrix, and subsequently to confined polymer chains in-between the nanometer-thick delaminated elementary clay layers. This leads to synergistic improvements in the composite properties, where the achieved properties are superior to those of the individual components. The fillers generally used for such composites are layered aluminosilicates, and most commonly montmorillonites (MMTs) from the family of aluminosilicates. The properties of the composites are directly affected by the filler volume fraction, the aspect ratio, alignment in the composite, and other geometric considerations.

The layered aluminosilicates such as MMT are plate-like particles and belong to the family of 2:1 phyllosilicates. A 2:1 layer consists of two tetrahedral silica sheets sandwiching an alumina octahedral sheet [11, 12]. The physical dimensions of one such layer may be $100 \mathrm{~nm}$ in diameter and $1 \mathrm{~nm}$ in thickness. Due to isomorphic substitutions in the octahedral and tetrahedral sheets, the layers have a net negative charge. The most common substitutions are $\mathrm{Al}^{3+}$ for $\mathrm{Si}^{4+}$ in the tetrahedral sheet and $\mathrm{Mg}^{2+}$ for $\mathrm{Al}^{3+}$ in the octahedral sheet. The negative charges are counterbalanced by the interlayer alkali or alkaline earth metal cations, and as a result of this the 2:1 layers are held together in stacks by electrostatic and van der Waals forces. Most of these inorganic minerals have

1) These studies were carried out at the Institute of Chemical and Bioengineering, Department of Chemistry and Applied Biosciences, ETH Zurich, 8093 Zurich, Switzerland. 
high energetic hydrophilic surfaces, which make them incompatible with the hydrophobic polymer matrices. MMTs are known to swell easily in water, and consequently can be delaminated in water to give nanosized platelets, the inorganic surface cations of which can then be exchanged with organic cations. An exchange of inorganic cations with organic cations renders the clay organophilic and hydrophobic, and lowers the surface energy of the clay layers. It then becomes possible for the organic polymer to diffuse between the clay layers and to delaminate the clay platelets to individual layers. This technology has been widely developed, as reported by Theng [13], Lagaly [14], Pinnavaia [15], and Giannelis [16]. Long-chain alkyl ammonium salts have been widely used for exchanging the inorganic cations because they increase the basal spacing of the clay to a large extent, apart from lowering the surface energy, which can further be helpful in achieving exfoliation of the clay layers in the polymer matrix. Figure 1.1 shows a representation of the surface modification process. Although, originally, the alkyl ammonium ions were the modifications conventionally used, in recent years more advanced surface modifications for the fillers have been developed; these include surface modifications with reactive groups, modifications with initiator molecules or with monomer molecules, and so on. Based on the basal plane spacing, it can also be predicted that either the alkyl chains lie flat on the silicate surface in monolayer, bilayer or pseudo-trimolecular arrangement, or radiate away from the surface giving paraffin-type geometry [17-20]. This may also help in predicting their possible interactions with the polymer matrix. It should be noted that the filler platelets are still partially polar, even after the surface modification with the long-chain alkyl ammonium ions, and therefore after drying would again form thick stacks of platelets joined
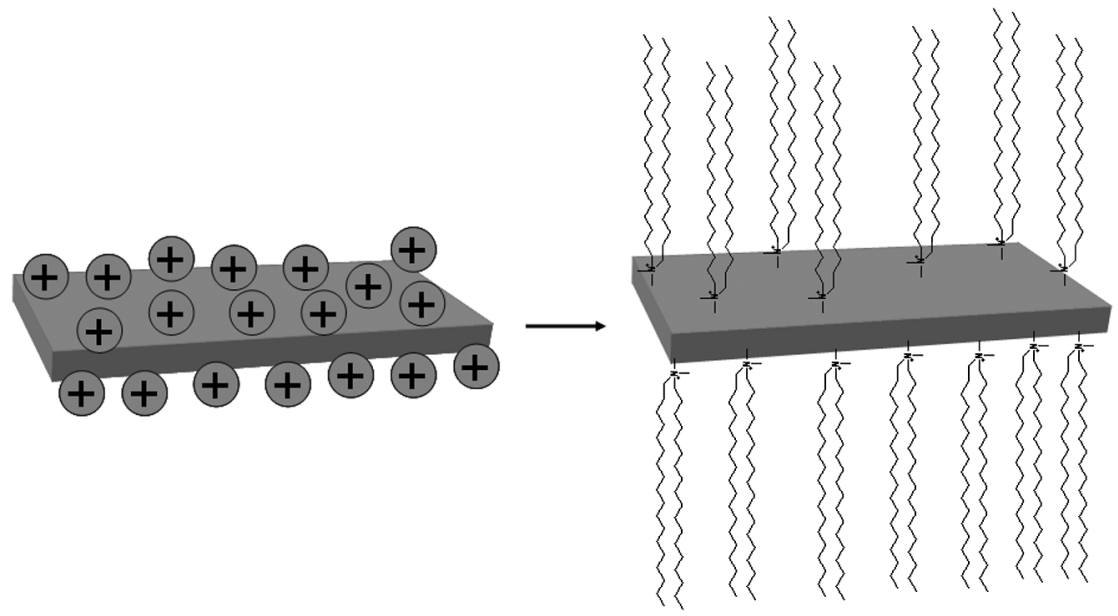

Figure 1.1 Surface modification of the aluminosilicate surface, which includes the exchange of cations present on the surface with long-chain alkyl ammonium ions. 
together by electrostatic forces. Thus, it is of immense importance to achieve the uniform and nanoscale dispersion of these platelets in the polymers matrix by the action of thermodynamic and kinetic forces. It is also possible to use the other varieties of aluminosilicates such as vermiculite or mica. These minerals differ from MMT in terms of their layer charge; this is high in mica and vermiculite, which makes them difficult to swell in water and hence to achieve complete surface modification. However, owing to the higher charges present per unit area as compared to the MMT surface, a greater degree of surface modification can be exchanged on the surface. This, in turn, leads to a more straight positioning of the modification molecules on the surface in the case of vermiculite and mica, thus creating a much higher basal plane spacing as compared to MMT for the same cation exchanged on the surface. The MMTs themselves also have wide range of layer charges or charge densities, depending on their source.

\section{2}

\section{Means of Synthesis and Microstructure}

Polymer nanocomposites have been generated in several different ways. In cases where the monomers are present in liquid form, a bulk polymerization of the monomer is often carried out in the presence of clay. This leads to much better interfacial contacts between the organic and inorganic phases, and the system does not suffer from the challenge of intercalation of high-molecular-weight polymer chains inside the clay interlayers. Otherwise, a solvent can also be used as the reaction medium in order to reduce the viscosity of the bulk medium and to distribute the heat more uniformly. In such cases, a solvent is chosen in which the polymer and monomer are soluble, and the solvent also is able to swell the clay. After polymerization and precipitation the precipitate can be collected and dried. Water may also be used as the reaction medium, while emulsion and suspension polymerization methods can also be used to generate polymer nanocomposites using monomers such as styrene and methylmethacrylate. In this case, the modified clay can be dispersed in the water phase by shearing; the emulsifier and monomer can then be added, followed by subsequent polymerization.

Melt compounding is the most commonly used approach for generating polymer nanocomposites. Here, high-molecular-weight polymers such as polypropylene (PP), polyethylene (PE) and polystyrene (PS) can be melted at high temperature, after which the modified clay powder is added to the melt. The filler is then kneaded and compounded thoroughly with the polymer melt in order to achieve a uniform dispersion and distribution of the filler. Although the melt compounding approach requires the use of a high temperature, this can sometimes cause concern with regards to surface modifications. Ammoniumbased surface modifications have an onset of degradation close to $200^{\circ} \mathrm{C}$, which is a common temperature used for melt compounding of the polymers. Any 
degradation of the surface modification may have a serious impact on the composite properties, and so should be avoided by using either a slightly lower temperature or more advanced thermally stable modifications. The time during which the organic inorganic phases are mixed at high temperature should also be optimized in order to minimize any thermal degradation of the ammonium modification.

The microstructure of the polymer nanocomposites is ideally classified as intercalated, exfoliated, and unintercalated or microcomposites. The composite microstructure is classified as exfoliated when the filler platelets are completely delaminated into their primary nanometer-scale size; moreover, the platelets should be far apart from each other so that the periodicity of the platelet arrangement is totally lost. This occurs when the electrostatic forces of interaction between the platelets have completely been overcome by the polymer chains. Figure 1.2 shows a series of transmission electron microscopy (TEM) images depicting the various morphologies of the polymer nanocomposite structures. Figure 1.2a represents the exfoliated morphology, where the black lines are the cross-section of the aluminosilicate platelets. The platelets can be seen as single and uniformly dispersed, although they are completely misaligned. On many occasions, bending and folding of the platelets has also been observed. For example, when a single (or occasionally more than one) extended polymer chain is intercalated into the clay interlayers, the periodicity of the clay platelets remains intact; such a microstructure is termed intercalated. Whilst this structure indicates that the organic-inorganic hybrid has been formed, the electrostatic forces of interaction between the clay platelets could not be totally dissolved. Figure $1.2 \mathrm{~b}$ shows the micrograph with intercalated platelets; here, the microstructure represents a well-ordered multilayer morphology consisting of alternate polymer and inorganic layers. Such a periodicity produces a signal in the X-ray diffractograms, such that the degree of intercalation can be quantified by comparison with the basal plane spacing of the modified filler. The exfoliated morphology does not generate any diffraction signal, owing to a loss of periodicity; an absence of any diffraction peak is taken as proof of the generation of completely exfoliated nanocomposites. Based on the interfacial interactions and mode of mixing of the organic and inorganic phases, it is possible that the two phases do not intermix at all, but rather a microcomposite or unintercalated composite is formed. Such a morphology is shown in Figure 1.2c. This type of structure is not a nanocomposite but, like conventional composites, will require a large amount of filler to achieve any significant improvement in the composite's properties, which otherwise could be achieved at much lower levels of filler in the case of nanocomposites. It should be noted that a classification of composite microstructure as either exfoliated or intercalated is unrealistic since, in reality, a variety of morphologies is generally present. Different extents of both intercalation and exfoliation are generally observed, whilst only a qualitative classification of morphology as more or less intercalated or exfoliated can generally be observed. 


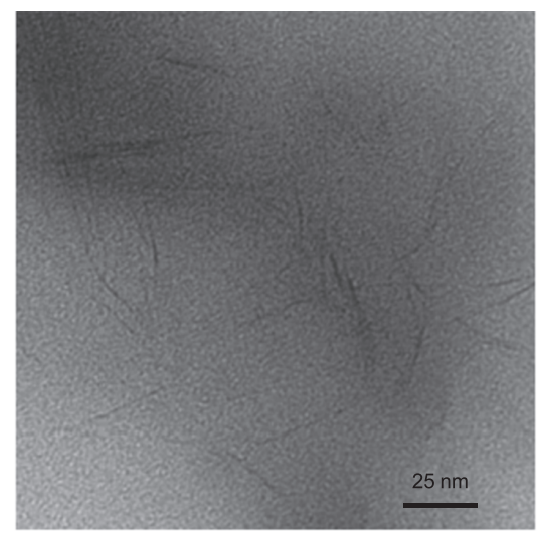

(a)

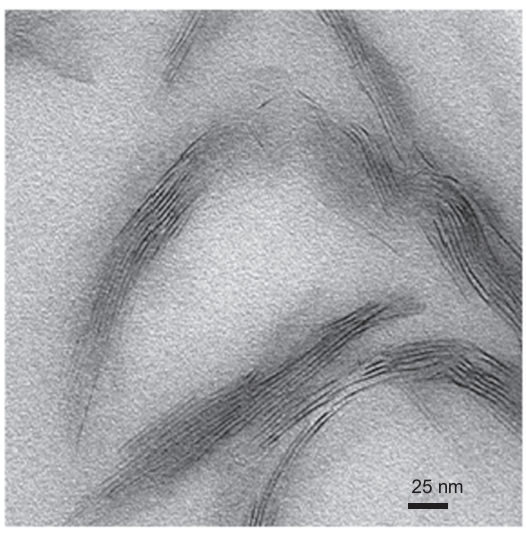

(b)

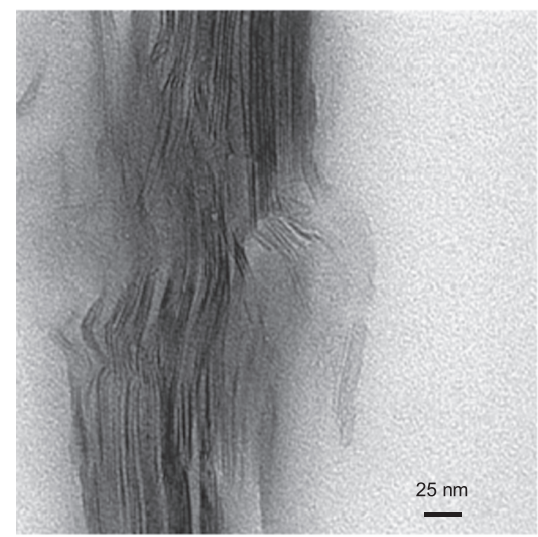

(c)

Figure 1.2 Transmission electron microscopy images indicating various possible morphologies in the composites as a function of the filler distribution. (a) Exfoliated; (b) Intercalated; (c) Unintercalated. 
1.3

\section{Importance of Thermogravimetric Analysis and X-Ray Diffraction for Filler and Nanocomposite Microstructure Characterization}

Both, XRD and TGA, are important techniques used to characterize the microstructure of nanocomposites. In general, TGA is used to assess the amount of organic matter exchanged on the clay surface during the surface modification process. High-resolution TGA can also be used to ascertain if there is presence of any excess of surface modification molecules present as a pseudo bilayer, but unbound to the surface. XRD is also used to quantify increases in basal plane spacing in the filler following surface modification, and also after composite generation. The increased basal plane spacing that occurs after surface exchange leads to information regarding the possible alignment of the modification molecules inside the clay interlayers, whereas the presence or absence of diffraction peaks in the composites is used to provide information concerning the microstructure of the composites. Although XRD also provides information relating to the amount of organic matter present in the clay interlayers, it cannot provide information on the excess of surface modification molecules present on the clay surface, as does TGA. Information relating to excess modification is very important, especially when the filler must be compounded with the polymer at high temperature, as any free modification present on the surface would have a much lower thermal degradation temperature and may impact negatively on the composite's properties [21]. Figure 1.3 shows a comparison of the XRD and TGA of the fillers, which were modified with octadecyltrimethylammonium, dioctadecyldimethylammonium and trioctadecylmethylammonium. The modifications differed in terms of the increasing number of octadecyl chains in the molecule. As shown in Figure 1.3a, the basal plane spacing of the filler increased as the chain density in the molecule increased. The cation-exchange capacity (CEC) of the clay also has a major impact on the basal plane spacing of the filler, with a low-CEC filler generally having a lower basal plane spacing as compared to a high-CEC counterpart. The fillers in Figure 1.3a had CEC-values of 680 and $880 \mu \mathrm{Eqg}^{-1}$. Figure 1.3b shows the TGA thermograms of the same fillers modified with the above-mentioned surface modifications, and using MMT with a CEC of $880 \mu \mathrm{Eqg}^{-1}$. The increased amount of organic matter was clearly visible in the TGA thermograms when the chain density was increased in accordance with the increase in basal plane spacing in the XRD. Yet, the TGA thermograms can provide additional information on the state of the surface of the MMTs, with those of the trioctadecylmethylammonium-modified MMT showing a sharp degradation peak at low temperature. This effect is due to a small amount of the ammonium modification being present as a pseudo-bilayer, and not bound ionically to the clay surface. As noted above, these molecules degrade at much lower temperatures than those which bind ionically to the clay surface; however, such information cannot be obtained from the X-ray diffractograms. Thus, it is very important to quantify the state of the filler surface by using a combination of high-resolution TGA and XRD. 


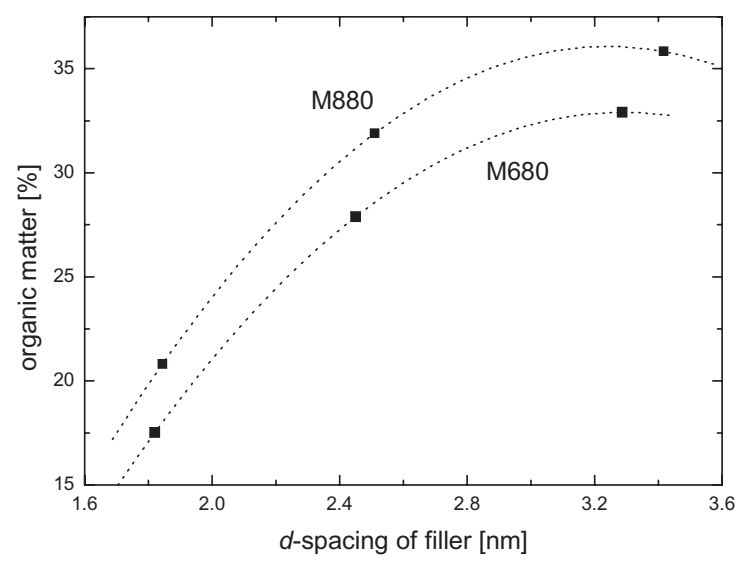

(a)

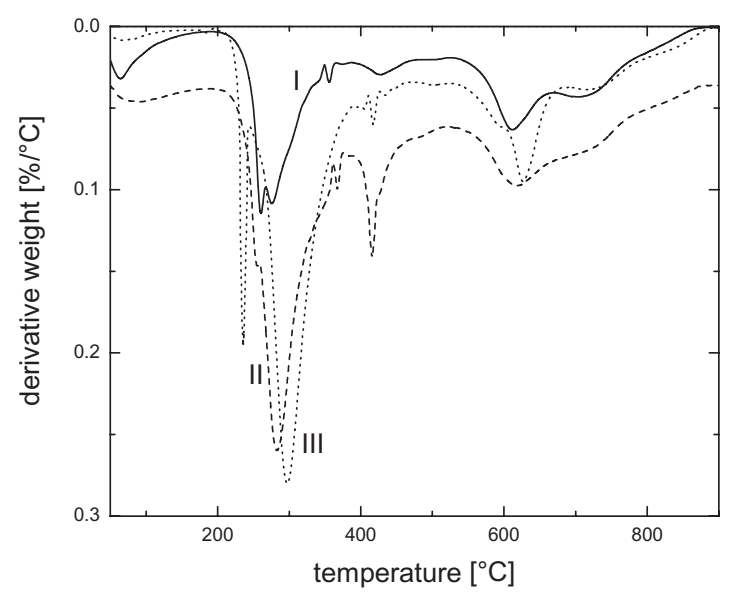

(b)

Figure 1.3 (a) Basal plane spacing in the montmorillonites with increasing chain density in the modification and as a function of filler cation-exchange capacity (CEC);

(b) Thermogravimetric analysis (TGA) thermograms of the filler with a CEC of $880 \mu \mathrm{Eqg}^{-1}$ modified with

(I) octadecyltrimethylammonium,

(II) dioctadecyldimethylammonium, and

(III) trioctadecylmethylammonium.

TGA can also be used to gain insights into the overall thermal performance of composites. As an example, Figure 1.4 shows the TGA thermograms of a pure polypropylene matrix (curve 1) and the MMT modified with dioctadecyldimethylammonium (curve 2). The TGA thermogram shown in curve 3 was obtained when these systems were formed into a composite with a 3 vol.\% filler content. With regards to the thermal behavior of the nanocomposite, a synergy between the composite components is clearly visible, as thermal degradation of the composite 


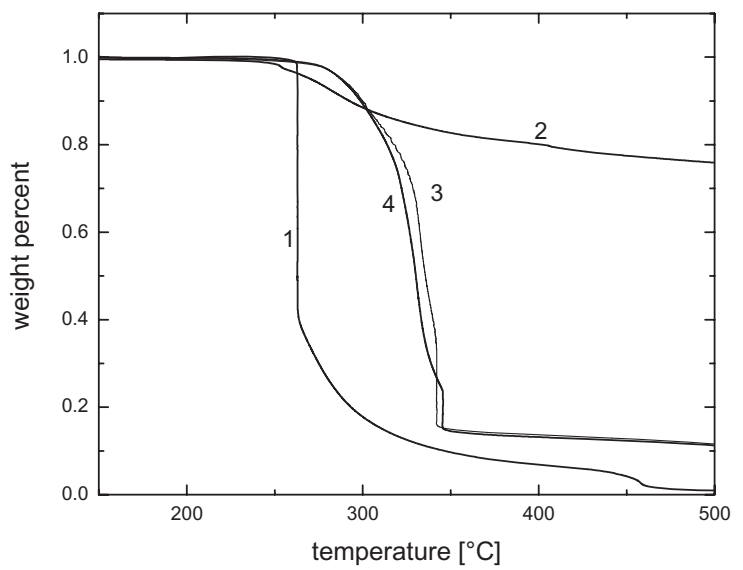

Figure 1.4 Thermogravimetric analysis thermograms of the (1) polypropylene (PP) matrix, (2) dioctadecyldimethylammoniummodified clay, (3) polypropylene composite

with 3 vol\% of clay of (2), and (4) polypropylene composite with 3 vol\% of clay of (2) and $4 \mathrm{wt} \%$ of PP-g-MA compatibilizer.

begins at a higher temperature than for any of the components. The TGA thermogram of the composite with $4 \mathrm{wt} \%$ polypropylene-grafted maleic anhydride compatibilizer is shown as curve 4 in Figure 1.4. As these compatibilizers have very low molecular weights, it is important to observe the thermal behavior of the composite in the presence of a compatibilizer. An examination of the TGA thermograms showed the thermal behavior of the composites to be similar in the presence or absence of compatibilizer, which indicated that the compatibilizer had no negative effect on thermal performance [22].

The intensity of X-ray diffractograms is generally taken as a measure of classifying the microstructure as either intercalated or exfoliated. For example, as shown in Figure 1.5, the intensity is seen to increase as the amount of filler in the composite is increased. It is thus notable that the composites become increasingly intercalated as the filler content rises, as a greater area under the curve would indicate a greater level of intercalated material. However, it should be noted that the X-ray signals are highly qualitative in nature and strongly influenced by the sample preparation and orientation of the platelets, as well as by defects present in the crystal structure of the MMTs. Thus, a classification of nanocomposite microstructure based only on the intensity may prove to be faulty. Neither does the presence of a diffraction signal in the diffractograms of the composite mean that $100 \%$ of the microstructure is intercalated; indeed, it is quite possible for there to be a significant amount of exfoliation present in the composite. Similarly, an absence of any diffraction signal does not guarantee complete exfoliation, as small or randomly oriented intercalated platelets may still be present in the composite. The X-ray diffractograms shown as examples in Figure 1.6a are of the MMT modified with benzyldibutyl(2-hydroxyethyl)ammonium, and its composite with epoxy. 


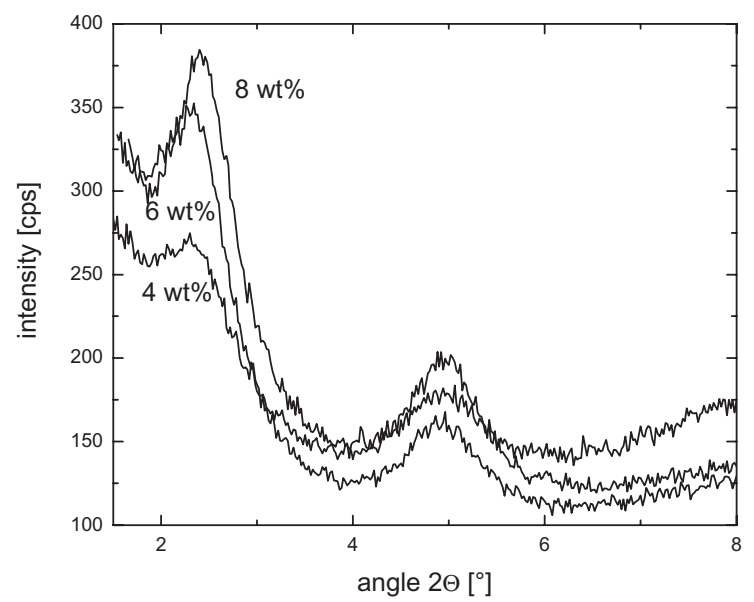

Figure 1.5 X-ray diffractograms of polyurethane nanocomposites containing different weight fractions of the filler.

Here, any increase in basal plane spacing of the filler after composite synthesis was minimal, while the diffraction signal of the filler in the composite material was quite strong in the diffractograms, and indicated an intercalated structure. However, when investigated with TEM, a large proportion of the filler was seen to exfoliated, and single platelets uniformly distributed in the polymer matrix (Figure 1.6b) [10]. It should be noted, therefore, that the XRD signals are qualitative in nature, and any idealized classification of the composite morphologies as interacted or exfoliated is entirely arbitrary.

\section{4}

\section{Polar and Nonpolar Polymer Systems}

Since the initial development of MMT/Nylon $6[2,23,24]$ nanocomposites by the research group at Toyota during the early 1990 s, investigations into reinforcing polymers by incorporating surface-treated layered aluminosilicates have been extensive. This technology of breaking the organically treated inorganic minerals into their nanoscale building blocks was successfully applied to polymer systems such as epoxies [10, 25-27], polyimides [28, 29], and polydimethylsiloxanes [30]. The intercalated and exfoliated nanocomposites achieved by this concept were found be very effective in improving the physical, mechanical and thermal properties of polymers at very low filler loadings as compared to the conventional composites. Conventional composites have the limitation that the clay tactoids form an unintercalated segregated phase in which the full potential of filler in improving the properties is never realized. For this reason, these composites require very 


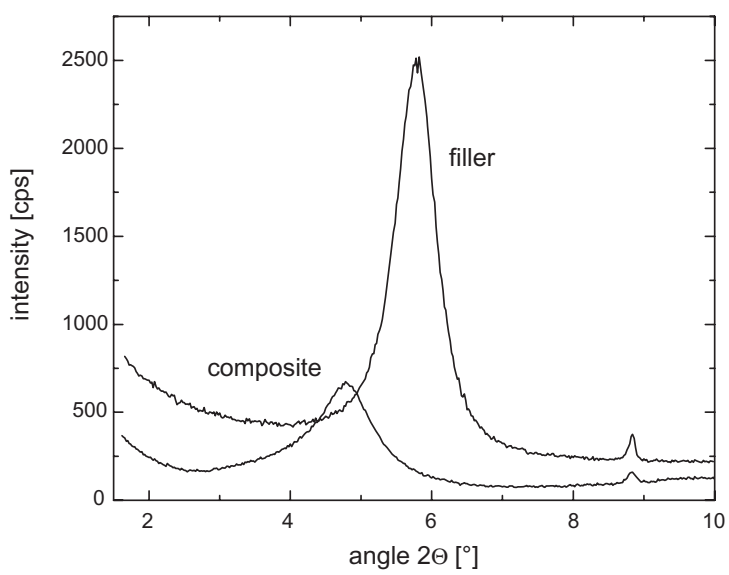

(a)

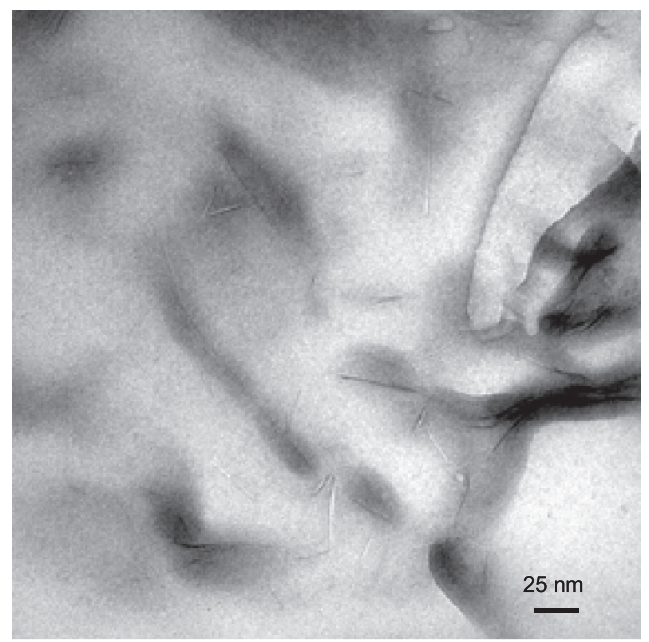

(b)

Figure 1.6 (a) X-ray diffractograms of benzyldibutyl (2-hydroxyethyl)ammonium-modified montmorillonite and its nanocomposites with epoxy polymer containing 3 vol\% filler;

(b) Transmission electron microscopy image of the epoxy nanocomposite.

high filler loadings in order to achieve impressive property improvements, but this makes them very bulky and opaque.

The mode of filler delamination in polar and nonpolar polymers has been observed to be very different. Polar polymers are generally observed to have more filler exfoliation as compared to nonpolar polymers, owing to the better match of polarity of polar polymers with the partially polar surface of the MMT. Thus, in the case of polar polymers, it is more likely the interfacial interactions between the organic and inorganic phases which lead to delamination of the filler. The 


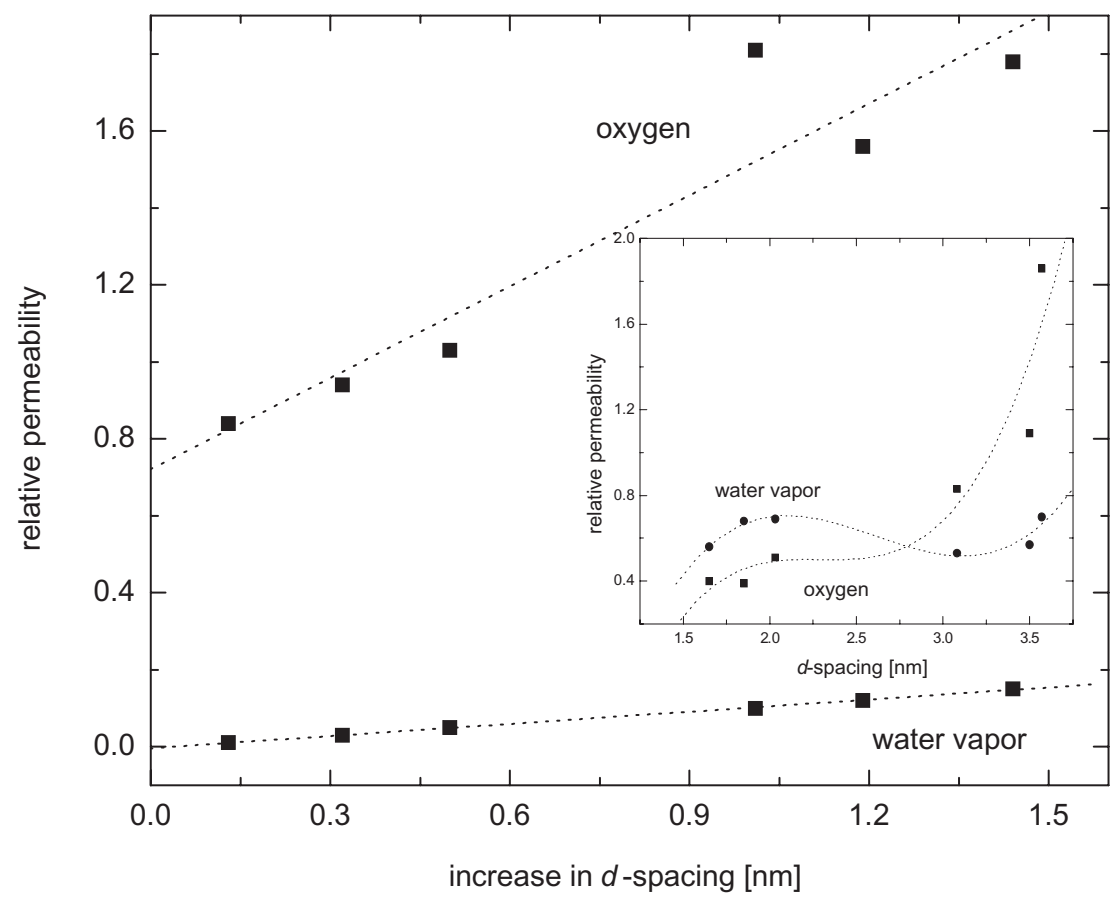

Figure 1.7 Permeation through the epoxy composites containing filler with different surface modifications as a function of filler basal plane spacing (see inset) or an increase in filler basal plane spacing in the composite.

basal plane spacing of the filler is not the prime factor to achieve exfoliation, as was also observed in Figure 1.6 for epoxy composites. The same notion is further reinforced in Figure 1.7, where oxygen and water vapor permeation though the epoxy nanocomposites is plotted as a function of the filler basal plane spacing in the composites, or as the increase in basal plane spacing of the filler after composite synthesis. In both cases, there is an indication of an increased permeation through the composites as the basal plane spacing is increased, which in truth should not be the case if the permeation were really influenced by the basal plane spacing of the filler. This confirms that it is not the basal plane spacing, but rather the exfoliated platelets that are generated, owing to the positive interfacial interactions which lead to improved composite properties in polar composites. There are generally also reactive modifications exchanged on the surface in such polymers, so that the polymer can be chemically tethered to the filler surface. As an example, benzyldibutyl(2-hydroxyethyl)ammonium was modified on the clay surface to generate epoxy nanocomposites, in the hope that the hydroxy groups of the modification would react chemically with the epoxy polymer chains. However, such bonds would be difficult to prove using infrared (IR) spectroscopy studies, as shown in Figure 1.8. The signal from the $\mathrm{OH}$ groups on the filler surface was observed at 


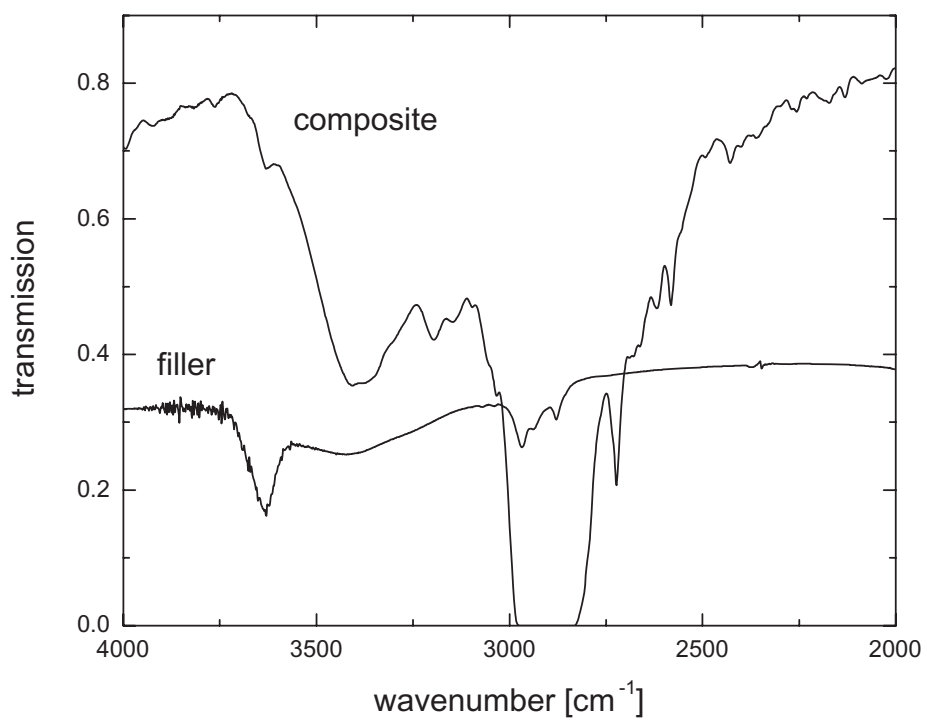

Figure 1.8 Infrared spectroscopy plots of the benzyldibutyl(2-hydroxyethyl)ammoniummodified clay, and its 3 vol\% epoxy nanocomposite.

approximately $3300 \mathrm{~cm}^{-1}$, an absence of which in the composite would confirm chemical tethering of the clay with the polymer matrix. However, there was a strong signal in this wavenumber range in the IR spectrum of the composite, owing to the generation of a large number of hydroxy groups on the polymer chains due to opening of the epoxy rings. Hence, whilst it is difficult to confirm the chemical reaction at the interface, the interfacial interactions in the studied systems were sufficiently positive to lead to a extensive delamination of the filler in the polymer matrix.

The nonpolar polymers, however, demonstrate different factors that affect delamination of the clay contained in them. In the absence of any positive interactions at the interface between the organic and inorganic phases, it is generally the basal plane spacing of the filler that determines the potential of the filler to be delaminated within the polymer matrix. A higher basal plane spacing in the filler leads to reduced electrostatic forces of interaction between the platelets, indicating that the platelets are more loosely held. This, in turn, increases the platelets' chances of exfoliation in the polymer matrices when mixed at high temperature in the compounder. This phenomenon is shown graphically in Figure 1.9, where PP composites with octadecyltrimethylammonium-, dioctadecyldimethylammoniumand trioctadecylmethylammonium-modified MMTs, using MMTs of two different CEC-values (680 and $880 \mu \mathrm{Eqg}^{-1}$ ) were analyzed for their tensile strength and oxygen permeation [31, 32]. On increasing the chain density, the basal plane spacing was seen to increase, the effect of which was also reflected in an increasing 


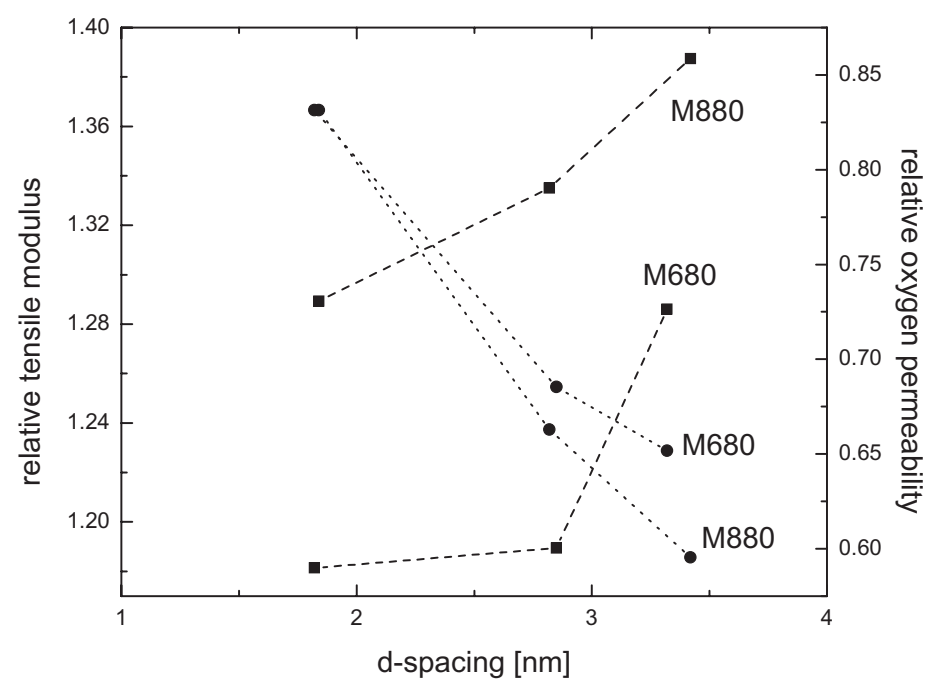

Figure 1.9 Improvements in the tensile modulus and oxygen permeation through polypropylene nanocomposites as a function of the cation-exchange capacity of the clay, and of the basal plane spacing of the modified clay.

value of the tensile modulus or a decreasing value of oxygen permeation. The data indicated clearly that an increased basal plane spacing in the filler rendered the platelets more susceptible to delamination, owing to reduced attractive forces among them.

It has also been shown important to ascertain the effect of a modified filler on the crystallinity of the polymer matrix. Occasionally, the filler may lead to changes in the crystallinity of the polymer, such that the composite properties become a result not only of the filler but also to crystallinity effects caused by the presence of the filler. The differential scanning calorimetric thermograms of the PP nanocomposites with octadecyltrimethylammonium-, dioctadecyldimethylammonium- and trioctadecylmethylammonium-modified MMTs are shown in Figure 1.10. Here, the thermograms were plotted as a comparison to the differential scanning calorimetry (DSC) thermogram of the pure PP matrix. The melting temperature and extent of crystallinity were observed to be unaffected by the change in the filler surface modification; this indicated that, under the conditions employed for synthesis of the composites, the modified filler had no effect on the crystallinity of the filler. However, Maiti et al. reported a decrease in spherulite size with an increase in clay content [33]. The presence of tactoids, owing to a poor dispersion of the filler, was also reported to cause a decrease in spherulite size [34]. Kodgire et al. reported that, in the presence of clay, PP showed an advanced crystallization and a fibrous morphology rather than the usual spherulite behavior [35]. Similarly, a decrease in crystallinity and an 


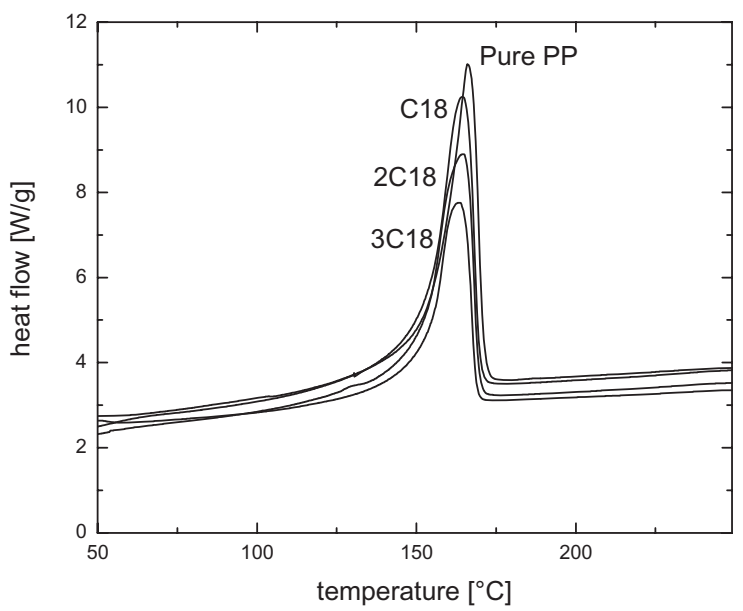

Figure 1.10 Differential scanning calorimetry dioctadecyldimethylammonium- (2C18), and thermograms of the polypropylene trioctadecylmethylammonium- (3C18) nanocomposites containing 3 vol\% of modified montmorillonites. octadecyltrimethylammonium- (C18),

increase in nucleus density was observed, owing to a nucleation effect of the clay platelets $[36,37]$.

\section{5}

\section{Advances in Filler Surface Modifications}

Apart from the conventional long-chain alkyl ammonium modifications, many developments have been introduced as surface modifications for inorganic fillers. Typically, these modifications have aimed at achieving a higher organophilization of the filler surface, in order to render them more susceptible to exfoliation when compounded with polymers (especially polyolefins). Surface reactions on the clay have been studied extensively so as to generate polymer chains and achieve much higher basal plane spacing values; this would otherwise be difficult to achieve via the exchange of preformed long chains, owing to problems of solubility and steric hindrance. The two forms of polymerization reaction are represented in Figure 1.11a: (i) polymerization "to" the surface, which is generally achieved by exchanging a monomer on the filler surface, followed by its polymerization with the external monomer; and (ii) polymerization "from" the surface, in which generally an initiator is bound ionically to the filler surface, which then is used to initiate the polymerization of an externally added monomer [38]. Other than these two options, a number of other possibilities of surface reactions exist, including surface esterification. Here, the reactive surface modifications are first exchanged on the clay surface, which can then be used to undergo simple esteri- 


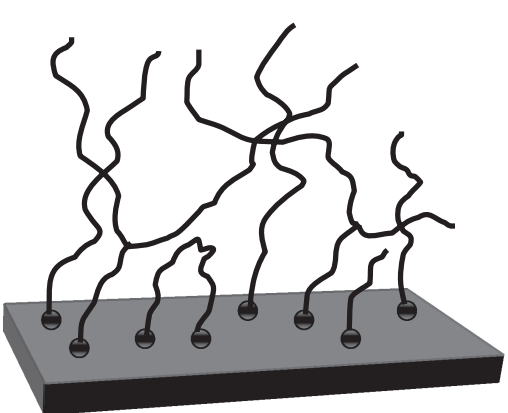

to

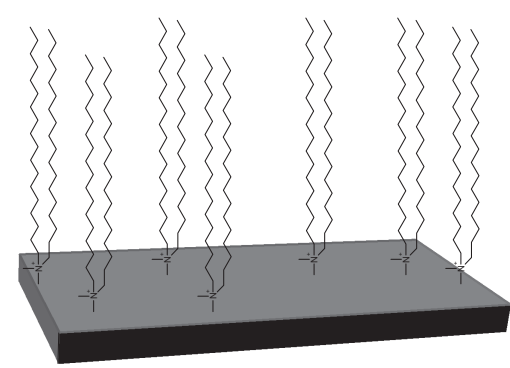

(b)

(a)

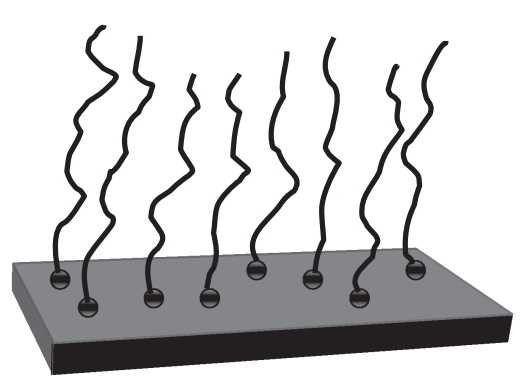

from

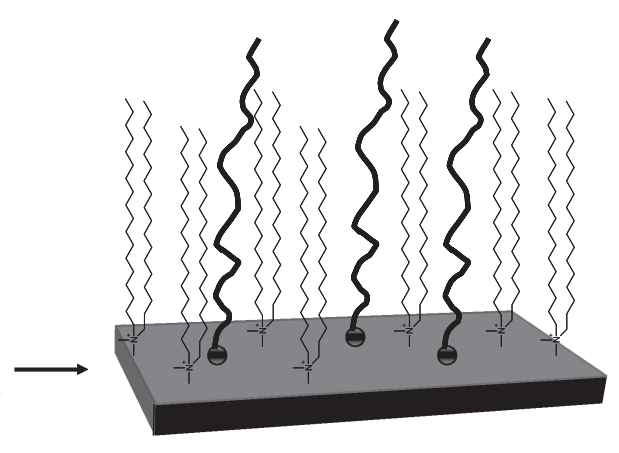

Figure 1.11 (a) Representation of the polymerization "to" the surface and polymerization "from" the surface; (b) Representation of physical adsorption onto the clay surface.

fication reactions leading to a higher basal plane spacing in the fillers [39]. The physical adsorption of organic molecules into vacant spaces on the clay surface after surface modification can also be used to achieve organophilization of the clay surface (Figure 1.11b). As the area available per cation is generally larger than the area of the cation exchanged on a clay surface, there will always be vacant spaces on the clay surface that can be targeted for occupation by organic molecules, with hydrogen bonds leading to much lesser electrostatic forces of interaction among the platelets [40].

\section{6}

\section{Prediction of Composite Properties}

The modeling of nanocomposite properties is important to achieve optimum improvements in composite properties, by optimizing the various factors that 


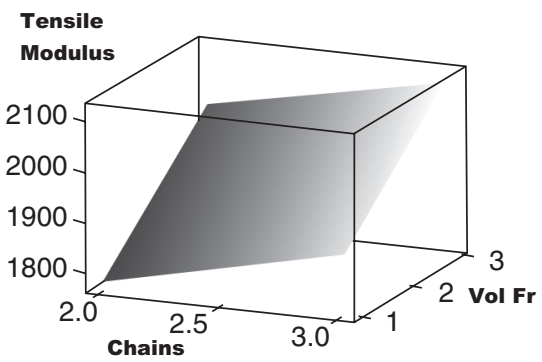

Figure 1.12 Prediction of tensile modulus of the polypropylene nanocomposites as a function of number of octadecyl chains in the ammonium modification and the inorganic volume fraction.

affect composite behavior. Although, in the past, many micromechanical models have been used traditionally to predict nanocomposite properties, these models have assumed the presence of: (i) a perfect alignment of the filler platelets in the composite; (ii) complete exfoliation of the filler; and (iii) interfacial adhesion [41-44]. These assumptions are not true, however, in the case of nanocomposites, and especially for polyolefins. Consequently, these models have been modified during recent years to incorporate the effects of incomplete exfoliation and misalignment [45-47]. In addition to these micromechanical models, several new models have been proposed that are based on factorial and mixture designs using a design of experiments methodology [48]. One such example is shown in Figure 1.12 , where the tensile modulus of the composites is predicted as a function of the octadecyl chain density in the surface modification and inorganic volume fraction. Such models do not include any unrealistic assumptions, and are thus more representative of the microstructure of the nanocomposites. These factorial designs can also help to quantify the interactions of the various factors (including volume fraction, chain density, and the CEC of the MMT) on each other, and to generate an entire spectrum of values of the composite property for different values of the factors.

A number of finite element models have also been developed to predict the properties of nanocomposites $[49,50]$. These models also incorporate the effects of incomplete exfoliation as well as filler misalignment, owing to the fact that these factors have significant effects on the composite properties. An example of this is shown in Figure 1.13, where the effect of platelet misalignment on the reduction of oxygen permeation through nanocomposite films is identified as a function of the aspect ratio of the platelets and the filler volume fraction. Although the reduction in oxygen permeation is much better when the platelets are completely aligned (Figure 1.13a), permeation is improved when the platelets are misaligned (Figure 1.13b). Hence, care must be taken when selecting a model used to predict a nanocomposite's properties; notably, the model should be capable of simulating the actual behavior of a nanocomposite material. 


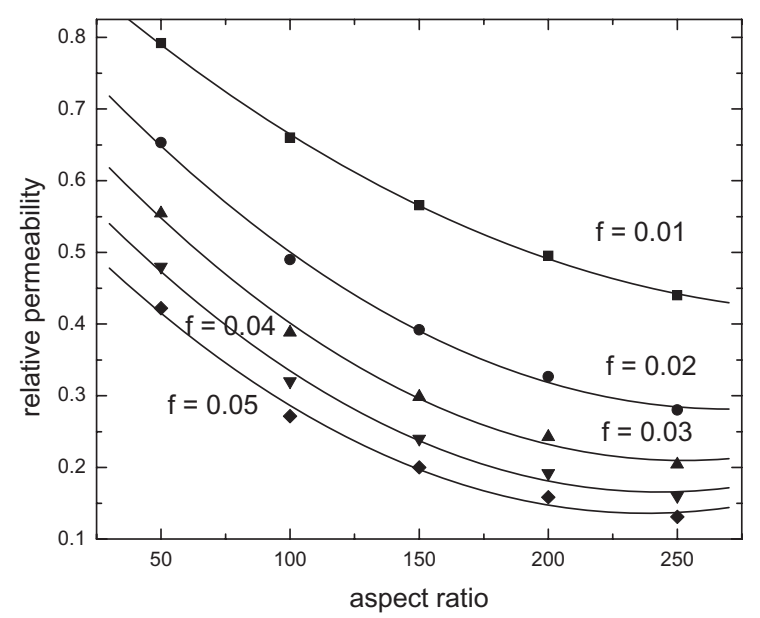

(a)

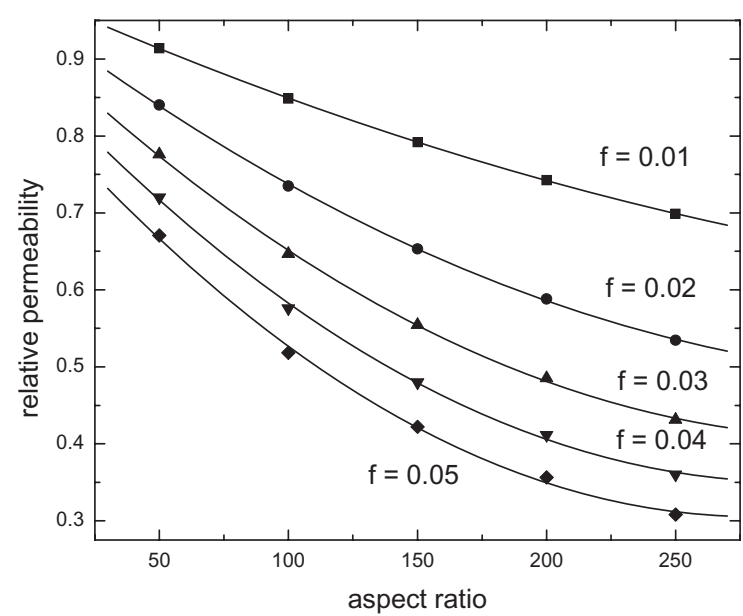

(b)

Figure 1.13 Predictions of reduction in oxygen permeation through the polymer nanocomposites as a function of aspect ratio and filler volume fraction when the filler platelets are (a) completely aligned and (b) completely misaligned [49].

\section{References}

1 Yano, K., Usuki, A., Okada, A., Kurauchi, T., and Kamigaito, O. (1993) J. Polym. Sci., Part A: Polym. Chem., 31, 2493.

2 Kojima, Y., Fukumori, K., Usuki, A., Okada, A., and Kurauchi, T. (1993) J. Mater. Sci. Lett., 12, 889.
3 Lan, T., Kaviratna, P.D., and Pinnavaia, T.J. (1994) Chem. Mater., 6, 573.

4 Lan, T. and Pinnavaia, T.J. (1994) Chem. Mater., 6, 2216.

5 LeBaron, P.C., Wang, Z., and Pinavaia, T.J. (1999) Appl. Clay Sci., 15, 11. 
6 Wang, Z. and Pinnavaia, T.J. (1998) Chem. Mater., 10, 3769.

7 Messersmith, P.B. and Giannelis, E.P. (1995) J. Polym. Sci., Part A: Polym. Chem., 33, 1047.

8 Yano, K., Usuki, A., and Okada, A. (1997) J. Polym. Sci., Part A: Polym. Chem., 35, 2289.

9 Osman, M.A., Mittal, V., Mobridelli, M., and Suter, U.W. (2003) Macromolecules, 36, 9851.

10 Osman, M.A., Mittal, V., Mobridelli, M. and Suter, U.W. (2004) Macromolecules, 37, 7250.

11 Bailey, S.W. (1984) Reviews in Mineralogy, Virginia Polytechnic and State University, Blacksburg.

12 Brindley, G.W. and Brown, G. (1980) Crystal Structures of Clay Minerals and Their X-Ray Identification (eds G.W. Brindley and G. Brown), Mineralogical Society, London.

13 Theng, B.K.G. (1974) The Chemistry of Clay-Organic Reactions, Adam Hilger, London.

14 Lagaly, G. (1986) Developments in Ionic Polymers (eds A.D. Wilson and H.J. Prosser), Elsevier Applied Science Publishers, London/New York.

15 Pinnavaia, T.J. (1983) Science, 220, 365.

16 Giannelis, E.P. (1996) Adv. Mater., 8, 29.

17 Lagaly, G. (1986) Solid State Ionics, 22, 43.

18 Lagaly, G. and Benecke, K. (1991) Colloid Polym. Sci., 269, 1198.

19 Osman, M.A., Seyfang, G., and Suter, U.W. (2000) J. Phys. Chem. B, 104, 4433.

20 Osman, M.A., Ernst, M., Meier, B.H., and Suter, U.W. (2002) J. Phys. Chem. B, 106, 653

21 Mittal, V. (2008) J. Comp. Mater., 42, 2829.

22 Mittal, V. (2008) J. App. Polym. Sci., 107, 1350.

23 Usuki, A., Kawasumi, M., Kojima, Y., Okada, A., Kurauchi, T., and Kamigaito, O. (1993) J. Mater. Res., 8, 1174.

24 Usuki, A., Kawasumi, M., Kojima, Y., Okada, A., Fukushima, Y., Kurauchi, T., and Kamigaito, O. (1993) J. Mater. Res., 8, 1179 .

25 Lee, A. and Lichtenhan, J.D. (1999) J. Appl. Polym. Sci., 73, 1993.
26 Becker, O., Cheng, Y.-B., Varley, R.J., and Simon, G.P. (2003) Macromolecules, 36, 1616.

27 Messersmith, P.B. and Giannelis, E.P. (1994) Chem. Mater., 6, 1719.

28 Wang, S., Ahmad, Z., and Mark, J.E. (1994) Proc. ACS, Div. Polym. Mater. Sci. Eng. (PMSE), 70, 305.

29 Kakimoto, M., Iyoku, Y., Morikawa, A., Yamaguchi, H., and Imai, Y. (1994) Polym. Prep., 35 (1), 393.

30 Burnside, S.D. and Giannelis, E.P. (1995) Chem. Mater., 7, 1597.

31 Osman, M.A., Mittal, V., and Suter, U.W. (2007) Macromol. Chem. Phys., 208, 68.

32 Mittal, V. (2007) J. Thermoplastic Comp. Mater., 20, 575

33 Maiti, P., Nam, P.H., Okamoto, M., Kotaka, T., Hasegawa, N., and Usuki, A (2002) Polym. Eng. Sci., 42, 1864.

34 Svoboda, P., Zeng, C., Wang, H., Lee, L.J., and Tomasko, D.L. (2002) J. Appl. Polym. Sci., 85, 1562.

35 Kodgire, P., Kalgaonkar, R., Hambir, S., Bulakh, N., and Jog, J.P. (2001) J. Appl. Polym. Sci., 81, 1786.

36 Gopakumar, T.G., Lee, J.A., Kontopoulou, M., and Parent, J.S. (2002) Polymer, 43, 5483.

37 Ma, J., Zhang, S., Qi, Z., Li, G., and Hu, Y. (2002) J. Appl. Polym. Sci., 83, 1978.

38 Mittal, V. (2007) J. Colloid Interface Sci., 314,141

39 Mittal, V. (2007) J. Colloid Interface Sci., 315,135

40 Mittal, V. and Herle, V. (2008) J. Colloid Interface Sci., 327, 295.

41 Kerner, E.H. (1956) Proc. Phys. Soc., B69, 808.

42 Hashin, Z. and Shtrikman, S. (1963) J. Mech. Phys. Solids, 11, 127.

43 Halpin, J.C. (1969) J. Comp. Mater., 3, 732.

44 Halpin, J.C. (1992) Primer on Composite Materials Analysis, Technomic, Lancaster.

45 van Es, M., Xiqiao, F., van Turnhout, J., and van der Giessen, E. (2001) Specialty Polymer Additives: Principles and Application (eds S. Al-Malaika, A.W. Golovoy, and C.A. Wilkie), Blackwell Science, Melden, CA, MA. 
46 Fornes, T.D. and Paul, D.R. (2003) Polymer, 44, 4993.

47 Brune, D.A. and Bicerano, J. (2002) Polymer, 43, 369-387.

48 Mittal, V. (2008) J. Thermoplastic Comp. Mater., 21, 9.
49 Bharadwaj, R.K. (2001) Macromolecules, 34, 9189.

50 Osman, M.A., Mittal, V., and Lusti, H.R. (2004) Macromol. Rapid Commun., 25, 1145. 
